\title{
Morfometría duodenal en pollos Cobb 500 suplementados con aceite esencial de orégano (Origanum vulgare $\mathrm{L}$ )
}

\author{
Duodenal morphometry in COBB 500 chickens supplemented with essential oil of \\ oregano (Origanum vulgare $\mathrm{L}$ )
}

\author{
Gustavo Adolfo Campozano-Marcillo ${ }^{1,3}$, Ernesto Antonio-Hurtado, Johnny Daniel \\ Bravo-Loor ${ }^{1}$, Carlos Aníbal Bulnes-Goicochea ${ }^{2}$, Valeria Lisseth Bazurto-Vera ${ }^{2}$, \\ Cristina Korin Solórzano- Macías ${ }^{2}$
}

\section{Resumen}

\begin{abstract}
El objetivo del estudio fue evaluar el efecto de la adición de aceites esenciales de orégano (AEO) en la dieta alimenticia sobre el comportamiento de la morfometría duodenal de pollos de engorde Cobb 500. Se utilizaron 400 pollos distribuidos en cinco tratamientos: suplementación con AEO $(100,200,300$ ppm) y dos tratamientos control (orégano comercial [250 ppm] y antibiótico promotor de crecimiento [300 ppm]), durante seis semanas, alimentados a voluntad con dietas formuladas semanalmente. El estudio morfométrico se realizó con ayuda de una cámara acoplada al microscópico mediante el software AmScope 3.0, objetivo de 4x, y campo de visión de $2000 \mu \mathrm{m}^{2}$ de área. La variable ancho proximal de las vellosidades fue significativamente mayor $(183 \mu \mathrm{m})$ en el tratamiento AEO $300 \mathrm{ppm}$ a los 21 días de edad y la variable profundidad proximal fue significativamente mayor $(256 \mu \mathrm{m})$ en el tratamiento AEO $300 \mathrm{ppm}$ a los 42 días $(\mathrm{p}<0.05)$. Se concluye que la adición de $\mathrm{AEO}$ en dosis de 300 ppm afectan positivamente la morfometría duodenal de pollos Cobb 500.
\end{abstract}

Palabras clave: aditivo natural para piensos, antibióticos, profundidad proximal, tracto gastrointestinal, vellosidades intestinales

\footnotetext{
${ }^{1}$ Carrera de Medicina Veterinaria, Escuela Superior Politécnica Agropecuaria de Manabi «Manuel Félix López» ESPAM-MFL, Manabi, Ecuador

${ }^{2}$ Escuela de Medicina Veterinaria y Zootecnia, Facultad de Ciencias Veterinarias, Universidad Técnica de Manabi (UTM), Manabi, Ecuador

${ }^{3}$ E-mail: gcampozanoregion4@gmail.com
}

Recibido: 21 de marzo de 2021

Aceptado para publicación: 30 de septiembre de 2021

Publicado: 22 de diciembre de 2021

CLos autores. Este artículo es publicado por la Rev Inv Vet Perú de la Facultad de Medicina Veterinaria, Universidad Nacional Mayor de San Marcos. Este es un artículo de acceso abierto, distribuido bajo los términos de la licencia Creative Commons Atribución 4.0 Internacional (CC BY 4.0) [https:// creativecommons.org/licenses/by/4.0/deed.es] que permite el uso, distribución y reproducción en cualquier medio, siempre que la obra original sea debidamente citada de su fuente original 
The aim of this study was to evaluate the effect of the addition of oregano essential oils (OEO) in the diet on duodenal morphometry of Cobb 500 broilers. In total, 400 chickens distributed in five treatments were used: supplementation with OEO $(100,200,300$ ppm) and two control treatments (commercial oregano [250 ppm] and growth-promoting antibiotic [300 ppm]), for six weeks, fed ad libitum with weekly formulated diets. The morphometric study was carried out with the help of a camera coupled to the microscope using AmScope 3.0 software, a 4x objective, covering an area of $2000 \mu \mathrm{m}^{2}$. The variable proximal width of the villi was significantly greater $(183 \mu \mathrm{m})$ in the OEO $300 \mathrm{ppm}$ treatment at 21 days of age and the variable proximal depth was significantly greater $(256 \mu \mathrm{m})$ in the OEO $300 \mathrm{ppm}$ treatment at 42 days $(\mathrm{p}<0.05)$. It is concluded that the addition of OEO in doses of 300 ppm positively affect the duodenal morphometry of Cobb 500 chickens.

Key words: natural feed additive, antibiotics, proximal depth, gastrointestinal tract, intestinal villi

\section{INTRODUCCIÓN}

La utilización de antibióticos como promotores de crecimiento en la elaboración de alimentos balanceados tiene por finalidad mejorar el rendimiento productivo y asegurar la salud animal: no obstante, esto ha generado el desarrollo de resistencia cruzada de ciertos microorganismos patógenos afectando la salud humana, debido al consumo de alimentos que contienen restos de antibióticos (Navarro y Larrañaga, 2012). Por ello, la Unión Europea prohibió el uso de antibióticos como método preventivo en la producción animal, generando la necesidad de reemplazarlos con aditivos naturales de origen vegetal, como los aceites esenciales, que realizan las misma o similares funciones que los promotores de crecimiento (EPC, 2013; Ardoino et al., 2018).

Los aceites esenciales que son compuestos aromáticos volátiles producto del metabolismo de las plantas (aditivos fitogénicos) son productos naturales bioactivos, empleados para incrementar el desempeño productivo e influir en el crecimiento y la salud de los animales (Piñón et al., 2015). La suplementación dietética de plantas medicinales aromáticas, hierbas, especias, sus extractos o aceites esenciales (AE) como productos fitogénicos ricos en compuestos antimicrobianos y antioxidantes puede mejorar la eficiencia en la utilización del alimento, así como el rendimiento y los beneficios económicos en la producción de pollos de engorde (Li et al., 2020).

La morfometría geométrica viene permitiendo realizar la evaluación cuanti y cualitativa de los cambios morfológicos (Toro et al., 2010). Así, el estudio morfométrico de la altura de las vellosidades, perímetro y profundidad de las criptas del duodeno se realiza a través del análisis de los cortes histológicos mediante un microscopio óptico acoplado a un sistema computarizado de captura de imágenes (Boaro et al., 2011).

El orégano (Origanum vulgare), pertenece a la familia Labiaceae. Es una planta herbácea aromática, utilizada por sus propiedades naturales y, que, además, posee antioxidantes, omega 3, hierro, magnesio, cobre y calcio, y aporta vitaminas como (B6) piridoxina, (B1) tiamina, (C) ácido ascórbico, (E) tocoferol y $(\mathrm{K})$ filoquinona, indispensa- 
Cuadro 1. Dieta experimental básica ${ }^{1}$ para pollos Cobb 5001 a 6 semanas

\begin{tabular}{lcccccc}
\hline \multirow{2}{*}{ Ingredientes } & \multicolumn{7}{c}{ Semana } \\
\cline { 2 - 7 } & 1 & 2 & 3 & 4 & 5 & 6 \\
\hline Maíz amarillo & 63.91 & 64.53 & 66.96 & 66.91 & 69.55 & 73.91 \\
Harina de soya 48\% & 28.25 & 27.64 & 26.25 & 25.87 & 22.25 & 19.25 \\
Aceite vegetal & 1.50 & 2.00 & 1.65 & 2.34 & 3.25 & 2.30 \\
Harina de pescado 65\% & 2.50 & 2.00 & 1.50 & 1.25 & 1.50 & 1.29 \\
Carbonato de calcio & 1.26 & 1.15 & 1.11 & 1.10 & 1.00 & 0.91 \\
Fosfato dicálcico & 1.48 & 1.50 & 1.29 & 1.25 & 1.00 & 0.79 \\
DL-Metionina 99\% & 0.14 & 0.15 & 0.14 & 0.14 & 0.12 & 0.12 \\
L-Lisina HCL 99\% & 0.12 & 0.16 & 0.15 & 0.15 & 0.18 & 0.17 \\
Premezcla Vit-Min Aves & 0.15 & 0.15 & 0.15 & 0.15 & 0.15 & 0.15 \\
Sal común & 0.36 & 0.37 & 0.38 & 0.39 & 0.39 & 0.40 \\
Bicarbonato de sodio & 0.35 & 0.37 & 0.44 & 0.46 & 0.62 & 0.73 \\
\hline
\end{tabular}

${ }^{1}$ Adicional a las materias primas utilizadas se añadió aceite esencial de orégano según los tratamientos, en función del contenido total del alimento

bles para el buen funcionamiento del organismo (Acevedo et al., 2013). El aceite esencial de orégano se ha propuesto como aditivo natural para su uso en pollos de engorde, teniendo su efecto principal en el tracto gastrointestinal, además de una acción moduladora sobre la microbiota intestinal (Betancourt et al., 2012).

El objetivo de este estudio fue evaluar el efecto de la adición de aceites esenciales de orégano (AEO) en la dieta alimenticia sobre el comportamiento de la morfometría duodenal de pollos de engorde de la línea genética Cobb 500.

\section{Materiales y Métodos}

\section{Localización y Duración}

El presente estudio se realizó en una nave convencional $(1.20 \mathrm{~m}$ de alto, $4 \mathrm{~m}$ de ancho y $12 \mathrm{~m}$ de largo) de producción de aves de corral, ubicado en un área destinada para la crianza de aves de la Carrera de Medicina Veterinaria, en la Escuela Superior Politécnica Agropecuaria de Manabí Manuel Félix López (ESPAM MFL), Ecuador. La crianza de las aves fue por 42 días, entre febrero y marzo de 2019. Las condiciones climáticas fueron: precipitación media anual de $911 \mathrm{~mm}$, temperatura media anual de $31{ }^{\circ} \mathrm{C}$, humedad relativa anual de $86.8 \%$, heliofania anual de 1109.8 h y una evaporación media anual de $499.6 \mathrm{~mm}$.

\section{Alimentación y Tratamientos}

El consumo de agua y alimento fue $a d$ libitum durante los primeros 14 días. Los pollos fueron asignados al azar a dos tratamientos a partir del día 15: Suministro de AEO y promotores de crecimiento.

El alimento fue ofrecido a voluntad con dietas formuladas por semanas (Cuadro 1) y de acuerdo con los requerimientos establecidos para pollos de engorde Coob 500 (CobbVantres, 2018). Para los cálculos de reque- 
rimientos nutricionales se utilizaron las ecuaciones sugeridas por Rostagno et al. (2017). El suministro de agua fue constante. El agua fue tratada con un neutralizador avícola llamado Neutralizador ${ }^{\circledR}$ (Pharmacy \& Nutrition) a razón de $1 \mathrm{ml} / 10 \mathrm{~L}$ de agua, además de cloro $(4 \mathrm{mg} / \mathrm{l})$.

Los tratamientos fueron:

- $\quad$ T01 $=$ Control $1-$ alimento balanceado comercial + (bacitracina) $25 \mathrm{ppm}$

- $\quad \mathrm{T} 02=$ Control 2 - alimento balanceado (Cuadro 1) + Orego-Stim (Chemical Pharm, Ecuador) 25 ppm

- $\quad \mathrm{T} 1=$ Alimento balanceado (Cuadro 1$)+$ aceite esencial de orégano $100 \mathrm{ppm}$

- $\quad \mathrm{T} 2=$ alimento balanceado (Cuadro 1) + aceite esencial de orégano $200 \mathrm{ppm}$

- $\quad \mathrm{T} 3=$ alimento balanceado (Cuadro 1$)+$ aceite esencial de orégano 300 ppm

\section{Animales}

Se utilizaron 400 pollos de un día de edad (unidades observacionales) de la línea Cobb 500, procedentes de la incubadora de la institución. Los pollos fueron colocados en 40 cubículos prefabricados de malla negra de polietileno $(1.25 \times 0.75 \times 0.9 \mathrm{~m})$. El primer día se tuvo una densidad de 30 pollos $/ \mathrm{m}^{2}$, pero cada tres días se retiraron 5 pollos $/ \mathrm{m}^{2}$ hasta quedar con 10 pollos $/ \mathrm{m}^{2}$. Cada cubículo estuvo constituido por 40 pollos que corresponden a cuatro repeticiones por tratamiento y 10 pollos por repetición, lo cual conformó una unidad experimental.

La limpieza y desinfección del galpón y los equipos se realizó dos semanas antes de la recepción de los pollos. Se utilizó agua y detergente para la limpieza general del galpón además de yodo $25 \%$ como un agente desinfectante mediante el método de aspersión con una dosis de $25 \mathrm{ml} / 1$ de agua.
Las calentadoras (focos 150 watts) fueron encendidos $6 \mathrm{~h}$ antes de la recepción de los pollos para obtener una temperatura óptima del galpón. Además, se usaron cortinas durante los primeros 14 días para evitar las corrientes de aire y mantener el calor. El control de la temperatura y humedad relativa se monitoreó tres veces al día mediante la ayuda de un higrómetro Vicks ${ }^{\circledR}$.

\section{Muestras}

Se seleccionaron 30 pollos (cinco por tratamiento) los días 21 y 42 . La técnica usada para la selección fue de tipo probabilístico, con muestreo aleatorio simple (Muestreo Simple al Azar), en donde cada sujeto tiene una probabilidad igual de ser seleccionado para el estudio (Walpole et al., 2012). El manejo de los animales se realizó siguiendo el Manual de Aplicabilidad de las Buenas Prácticas Avícolas de Agrocalidad del Ecuador (Vizcaíno et al., 2013).

Los animales se sacrificaron mediante electroshock y desangrado. Luego se incidió la cavidad abdominal y se expuso el tracto gastrointestinal, para realizar el análisis morfopatológico a fin de identificar alteraciones macroscópicas. Finalmente, se tomaron muestras del duodeno por medio de cortes transversales de $1 \mathrm{~cm}$ de la región proximal, medial y distal (total: 180 muestras). Las muestras fueron fijadas con solución tamponada de formol al 10\% y se colocaron en un cooler para el transporte hacia el laboratorio de la Facultad de Ciencias Veterinarias de la Universidad Técnica de Manabí (UTM).

\section{Estudio Morfométrico}

Las muestras fueron sometidas al procesamiento histológico estándar, y se realizaron cortes transversales de $3 \mathrm{~mm}$. Las láminas fueron observadas en un microscopio óptico trinocular estándar (Optika B-383PLi, 
Italia). La captura de las imágenes de las láminas histológicas se realizó con ayuda de una cámara acoplada al microscopio (AmScope MU1000) mediante el software AmScope 3.0, utilizando el objetivo de 4x, con un campo de visión de $2000 \mu \mathrm{m}^{2}$ de área. Se registraron las siguientes variables de las vellosidades intestinales: ALP: Altura proximal; ALM: Altura medial; ALD: Altura distal; AP: Ancho proximal; AM: Ancho medial; AD: Ancho distal; PP: Profundidad proximal; PM: Profundidad medial y PD: Profundidad distal. Todas las mediciones se tomaron en milímetros ( $\mathrm{mm}$ ) y posteriormente se las transformó a micrómetros $(\mu \mathrm{m})$ para trabajar con datos de mayor precisión.

La altura de las vellosidades se mide desde el área basal, coincidiendo con la porción superior de las criptas hasta su vértice. Esta medida permite además determinar el número de vellosidades presentes un área determinada. La altura de las criptas se mide desde la base a la región de transición de la cripta (Pelicano et al., 2003).

\section{Análisis Estadístico}

Se aplicaron las pruebas de ShapiroWilk y Levene para probar la normalidad de los datos y homogeneidad de la varianza de las variables en estudio. Se utilizó el análisis de varianza de una vía en un diseño completamente aleatorizado utilizando un paquete de software estadístico con el procedimiento GLM del SAS v. 9.4 (2013). Se utilizó la prueba de Tukey para detectar significancia $(p<0.05)$ entre las medias. La prueba de rango múltiple de Dunnett se aplicó como una prueba post-hoc para las diferencias entre la media de cada nivel de factor (tratamiento) y la media de un grupo de control con intervalo de confianza al 95\% ( $<<0.05)$. Los resultados se presentaron como media \pm error estándar de la media.

\section{Resultados y Discusión}

\section{Variables Morfométricas}

En los resultados de la morfometría de las vellosidades intestinales en el periodo de 21 días (cuadros 2 y 3), se encontró diferencia significativa en la variable ancho proximal, a favor del tratamiento con AEO 200 ppm $(183 \mu \mathrm{m})$.

Estudios como el de Macari et al. (2002) afirman que las vellosidades del duodeno son más largas que las del yeyuno e íleon, independientemente de la edad; asimismo, cuanto más lejos del proventrículo y de la molleja, más pequeñas serán las vellosidades y menos los cambios morfológicos. Es por esto que el presente estudio se realizó con muestras de duodeno, por ser la sección del intestino delgado con mayor capacidad de digestión y absorción, tal como lo ha indicado Boaro et al. (2011).

Betancourt et al. (2012) obtuvieron resultados similares al analizar el efecto de los aceites esenciales de orégano en pollos de engorde sobre el ancho de las vellosidades a la misma edad, obteniendo una diferencia a favor de $4.70 \%$, siendo un indicador positivo para la buena absorción de nutrientes, y estimulación del sistema inmune (Kondo, 2003).

A los 42 días se pudo notar que existen diferencias estadísticas para la variable profundidad proximal, siendo el tratamiento AEO $300 \mathrm{ppm}$ el de mayor efecto (cuadros 4 y 5 ; $\mathrm{p}<0.05)$. El promedio encontrado $(256 \mu \mathrm{m})$ es similar al reportado por Madrid et al. (2018), quienes encontraron mayor profundidad y altura de las vellosidades intestinales del duodeno al incluir tres niveles diferentes de AEO en pollos de engorde Cobb 500. En tal sentido, la morfología intestinal se mejora por efecto del orégano sobre las toxinas. Se 
Cuadro 2. Promedios y error estándar en variables morfométricas del duodeno de pollos Cobb 500 a los 21 días de edad

\begin{tabular}{cccccccc}
\hline & \multicolumn{5}{c}{ Tratamientos } & Valor \\
\cline { 2 - 6 } & Control 1 & Control 2 & AEO 100 ppm & AEO 200 ppm & AEO 300 ppm & p \\
\hline ALP & $1686.0 \pm 375.96$ & $1670.0 \pm 269.17$ & $1650.0 \pm 318.58$ & $1537.0 \pm 402.97$ & $1725.0 \pm 336.99$ & 0.77 \\
ALM & $1737.0 \pm 325.63$ & $1610.0 \pm 263.31$ & $1637.0 \pm 380.77$ & $1514.0 \pm 377.52$ & $1685.0 \pm 419.5$ & 0.53 \\
ALD & $1718.0 \pm 374.77$ & $1637.0 \pm 320.28$ & $1738.0 \pm 292.13$ & $1607.0 \pm 300.33$ & $1551.0 \pm 403.03$ & 0.60 \\
AP & $132.0^{\mathrm{b}} \pm 26.64$ & $136.0^{\mathrm{b}} \pm 29.35$ & $135.0^{\mathrm{b}} \pm 25.19$ & $183.0^{\mathrm{a}} \pm 69.64$ & $149.0^{\mathrm{b}} \pm 36.5$ & 0.01 \\
AM & $134.0 \pm 26.64$ & $142.0 \pm 29.49$ & $150.0 \pm 40.32$ & $175.0 \pm 59.65$ & $136.0 \pm 31.28$ & 0.53 \\
AD & $137.0 \pm 26.8$ & $143.0 \pm 38.92$ & $150.0 \pm 70.79$ & $150.0 \pm 36.01$ & $135.0 \pm 25.05$ & 0.97 \\
PP & $309.0 \pm 69.71$ & $292.0 \pm 43.45$ & $339.0 \pm 60.71$ & $297.0 \pm 64.87$ & $306.0 \pm 35.14$ & 0.21 \\
PM & $300.0 \pm 62.79$ & $319.0 \pm 61.41$ & $320.0 \pm 53.8$ & $322.0 \pm 54.47$ & $329.0 \pm 65.65$ & 0.84 \\
PD & $307.0 \pm 49.54$ & $294.0 \pm 49.48$ & $313.0 \pm 57.52$ & $290.0 \pm 48.31$ & $360.0 \pm 66.63$ & 0.19 \\
\hline
\end{tabular}

ALP: Altura proximal, ALM: Altura medial, ALD: Altura distal, AP: Ancho proximal, AM: Ancho medial, AD: Ancho distal, PP: Profundidad proximal, PM: Profundidad medial, PD: Profundidad distal

a,b Tratamiento con superíndice diferente son estadísticamente diferentes $(p<0.05)$

${ }^{1}$ Control 1: balanceado comercial + bacitracina 25 ppm; Control 2: alimento formulado (Cuadro 1) + Orego-Stim; AEO 10, AE= 200, AEO 200 ppm = alimento formulado (Cuadro 1) + aceite esencial de orégano 100. 200 o 300 ppm, respectivamente.

Cuadro 3. Ancho proximal de las vellosidades intestinales del duodeno de pollos Cobb 500 a los 21 días de edad (Test Dunnett)

\begin{tabular}{llcllc}
\hline \multicolumn{2}{c}{ Tratamientos } & $p$-valor & \multicolumn{2}{c}{ Tratamientos } & $p$-valor \\
\hline Control 1 & Control 2 & 0.99 & Control 2 & Control 1 & 0.99 \\
Control 1 & AEO 100 ppm & 0.99 & Control 2 & AEO 100 ppm & 1.00 \\
Control 1 & AEO 200 ppm & 0.01 & Control 2 & AEO 200 ppm & 0.02 \\
Control 1 & AEO 300 ppm & 0.63 & Control 2 & AEO 300 ppm & 0.82 \\
\hline
\end{tabular}

${ }^{1}$ Control 1: balanceado comercial + bacitracina 25 ppm; Control 2: alimento formulado (Cuadro 1) + Orego-Stim; AEO 10, AE= 200, AEO 200 ppm = alimento formulado (Cuadro 1) + aceite esencial de orégano 100.200 o $300 \mathrm{ppm}$, respectivamente

sabe que las toxinas bacterianas tienen efectos negativos sobre la morfología intestinal (Samadian et al., 2013).

No hubo diferencias significativas con respecto a las demás variables en estudio, similar al reporte de Botsoglou et al. (2010), quienes no detectan efectos destacables en la histomorfometría del intestino delgado en pollos con dietas que incluyen aceites esenciales de orégano. Por otro lado, Ayala et al. (2006) y Shiva et al. (2012), utilizando orégano como promotor de crecimiento tampoco encontraron diferencias favorables en parámetros productivos como ganancia de peso, consumo de alimento y conversión alimenticia en comparación a los antibióticos promotores de crecimiento. 
Cuadro 4. Promedios y error estándar en variables morfométricas del duodeno de pollos Cobb 500 a los 42 días de edad

\begin{tabular}{ccccccc}
\hline & \multicolumn{5}{c}{ Tratamientos $^{1}$} & Valor \\
\cline { 2 - 6 } & Control 1 & Control 2 & AEO 100 ppm & AEO 200 ppm & AEO 300 ppm & p \\
\hline ALP & $1282.0 \pm 197.03$ & $1325.0 \pm 216.17$ & $1395.0 \pm 278.61$ & $1287.0 \pm 390.90$ & $1234.0 \pm 340.63$ & 0.44 \\
ALM & $1015.0 \pm 170.17$ & $1356.0 \pm 177.43$ & $1162.0 \pm 262.90$ & $1458.0 \pm 280.94$ & $1248.0 \pm 328.55$ & 0.21 \\
ALD & $1082.0 \pm 135.00$ & $1276.0 \pm 207.75$ & $1150.0 \pm 329.26$ & $1330.0 \pm 245.65$ & $1184.0 \pm 336.02$ & 0.69 \\
AP & $114.0 \pm 25.77$ & $115.0 \pm 29.08$ & $123.0 \pm 36.31$ & $129.0 \pm 37.56$ & $129.0 \pm 34.16$ & 0.63 \\
AM & $105.0 \pm 26.54$ & $115.0 \pm 23.17$ & $116.0 \pm 31.03$ & $124.0 \pm 35.07$ & $112.0 \pm 26.39$ & 0.53 \\
AD & $88.0 \pm 15.32$ & $118.0 \pm 22.37$ & $127.0 \pm 36.37$ & $118.0 \pm 35.40$ & $106.0 \pm 24.27$ & 0.10 \\
PP & $202.0 \pm 30.66$ & $190.0 \pm 28.65$ & $209.0 \pm 44.99$ & $244.0 \pm 64.68$ & $256.0^{\mathrm{a}} \pm 60.78$ & $* 0.02$ \\
PM & $177.0 \pm 25.39$ & $195.0 \pm 39.13$ & $223.0 \pm 42.66$ & $232.0 \pm 43.03$ & $213.0 \pm 52.20$ & 0.20 \\
PD & $182.0 \pm 23.58$ & $211.0 \pm 38.41$ & $204.0 \pm 42.14$ & $272.0 \pm 54.35$ & $220.0 \pm 41.32$ & 0.09 \\
\hline
\end{tabular}

ALP: Altura proximal, ALM: Altura medial, ALD: Altura distal, AP: Ancho proximal, AM: Ancho medial, AD: Ancho distal, PP: Profundidad proximal, PM: Profundidad medial, PD: Profundidad distal

a,b Tratamiento con superíndice diferente son estadísticamente diferentes $(p<0.05)$

${ }^{1}$ Control 1: balanceado comercial + bacitracina $25 \mathrm{ppm}$; Control 2: alimento formulado (Cuadro

1) + Orego-Stim; AEO 10, AE= 200, AEO 200 ppm = alimento formulado (Cuadro 1) + aceite esencial de orégano 100. 200 o 300 ppm, respectivamente

Cuadro 5. Ancho proximal de las vellosidades intestinales del duodeno de pollos Cobb 500 a los 42 días de edad (Test Dunnett)

\begin{tabular}{llcllc}
\hline \multicolumn{2}{c}{ Tratamientos } & $p$-valor & \multicolumn{2}{c}{ Tratamientos } & $p$-valor \\
\hline Control 1 & Control 2 & 0.95 & Control 2 & Control 1 & 0.95 \\
Control 1 & AEO 100 ppm & 0.99 & Control 2 & AEO 100 ppm & 0.80 \\
Control 1 & AEO 200 ppm & 0.20 & Control 2 & AEO 200 ppm & 0.07 \\
Control 1 & AEO 300 ppm & 0.06 & Control 2 & AEO 300 ppm & 0.02 \\
\hline
\end{tabular}

${ }^{1}$ Control 1: balanceado comercial + bacitracina 25 ppm; Control 2: alimento formulado (Cuadro 1) + Orego-Stim; AEO 10, AE= 200, AEO 200 ppm = alimento formulado (Cuadro 1) + aceite esencial de orégano 100.200 o $300 \mathrm{ppm}$, respectivamente

\section{Conclusiones}

El empleo de 300 ppm de aceite esencial de orégano (AEO) en la dieta de los po llos de engorde, en reemplazo de antibióticos promotores de crecimiento puede servir para mejorar los parámetros morfométricos del intestino delgado y el estado de salud de las aves. 


\section{Literatura Citada}

1. Ayala L, Martínez M, Acosta D, Hernández O. 2006. Una nota acerca del efecto del orégano como aditivo en el comportamiento productivo de pollos de Ceba. Cuban J Agr Sci 4: 455-458.

2. Acevedo D, Navarro M, Monroy L. 2013. Composición química del aceite esencial de hojas de orégano (Origanum vulgare). Información Tecnológica 24: 43-48. doi: 10.4067/S0718-07642013000400005

3. Ardoino SM, Toso RE, Álvarez HL, Mariani EL, Cachau PD, Mancilla MV, Oriani DS. 2018. Antimicrobial as growth promoters (AGP) in poultry balanced feed: use, bacterial resistance, new alternatives and replacement options. Cienc Vet 19: 50-66. doi: 10.19137/cienvet-20171914

4. Betancourt L, Ariza C, Afanador G. 2012. Effects of supplementation with oregano essential oil on ileal digestibility, intestinal histomorphology, and performance of broiler chickens. Rev Colomb Cienc Pec 25: 240-251.

5. Boaro M, Fernandes B, Mendes A, Milbradt E, Martins B, Aguiar E. 2011. Morfometria da mucosa do duodeno de frangos de corte suplementados com melhoradores de desempenho. En: XXII Congresso Latino-Americano de Avicultura. Buenos Aires, Argentina.

6. Botsoglou NA, Florou-Paneri P, Christaki E, Fletouris DJ, Spais AB. 2010. Effect of dietary oregano essential oil on performance of chickens and on iron-induced lipid oxidation of breast, thigh and abdominal fat tissues. Brit Poultry Sci 43: 223-230. doi: 10.1080/ 00071660120121436. PMID: 12047086.

7. Cobb-Vantres. 2018. Broiler Cobb-500. Broiler performance \& nutrition supplement. https://www.cobbvantress.com/-assets/Cobb-Files/productguides/ bdc20a5443/70dec630-0abf-11e99c88c51e407c53ab.pdf
8. [EPC] European Parliament and Council. 2003. Regulation No 1831/ 2003 of the European Parliament and of the Council of $22^{\text {nd }}$ September 2003 on additives for use in animal nutrition. Official Journal of the European Union. L268/36.

9. Kondo N. 2003. Estudo das características morfométricas de diferentes regiões do intestino delgado e índices zootécnicos em quatro linhagens de frangos de corte. Tesis de Doutor em Medicina Veterinária. Botucatu, Brasil: Universidade Estadual Paulista. $107 \mathrm{p}$.

10. Li-Zhi Jin, Yueming Dersjant-Li, Ilias Giannenas. 2020. Application of aromatic plants and their extracts in diets of broiler chickens. In: Feed additives. Academic Press. p 159-185.

11. Macari M, Furlan R, Gonzales E. 2002. Fisiologia aviairia aplicada a frangos de corte. Jaboticabal, Brazil. 375 p.

12. Madrid T, Lopez A, Parra J. 2018. La ingesta de aceite esencial de orégano (Lippia origanoides) mejora la morfología intestinal en Broilers. Arch Zootec 67: 70-76.

13. Navarro AA, Larrañaga MR. 2012. Residuos de medicamentos de uso veterinario: Toxicología alimentaria. En: Fernández AM, Jiménez MR (eds), Toxicología alimentaria. Ed Díaz de Santos. p 393-396.

14. Pelicano E, Souzaa P, Obab A, Norkus E, Kodawara L, Azevedo T. 2003. Morfometria e ultraestrutura da mucosa intestinal de frangos de corte alimentados com dietas contendo diferentes probióticos. Rev Port Cienc Vet 98: 1252256.

15. Piñón JR, Monterrubio AL, Meléndez $L A$, Martínez AC, Rojo AD, Palma NG, Vásquez RS. 2015. Efecto del aceite esencial de orégano en el rendimiento y las propiedades fisicoquímicas y microbiológicas de la carne de pollo. Investigación y Ciencia 65: 5-11. 
16. Rostagno HS, Teixeira Albino LF, Hannas MI, Lopes Donzele J, Sakomura NK, Perazzo FG, Oliveira Brito C. 2017. Tabelas brasileiras para aves e suínos. Composição de alimentos e exigências nutricionais. $4^{\circ}$ ed. Viçosa, Brazil: Universidade Federal de Viçosa. $488 \mathrm{p}$.

17. Samadian F, Zeinoaldini S, Towhidi $A$, Torshizi MAK, Pirasaraei $Z A$, Gholamzadeh P. 2013. Evaluation of some phytogenic feed additives in growing chicks diet. Int J Agric Res Rev 3: 35-43.

18. SAS Institute Inc. 2013. SAS/ ACCESS ${ }^{\circledR} 9.4$ Interface to ADABAS: Reference. Cary, NC: SAS Institute Inc.

19. Shiva C, Bernal S, Sauvain M, Caldas J, Kalinowski J, Falcón N, Rojas R. 2012. Evaluación del aceite esencial de orégano (Origanum vulgare) y extracto deshidratado de jengibre (Zingiber officinale) como potenciales promotores de crecimiento en pollos de engorde. Rev Inv Vet Perú 23: 160-170. doi: 10.15381/rivep.v23i2.896

20. Toro M, Manríquez, G, Suazo I. 2010. Morfometría geométrica y el estudio de las formas biológicas: de la morfología descriptiva a la morfología cuantitativa. Int J Morphol 28: 977-990. doi: 10.4067/ S0717-95022010000400001

21. Vizcaino D, Betancourt R, Espinosa M, Egas V, Orellana J, Guevara E, Nogales H. 2013. Manual de aplicabilidad de las buenas prácticas avícolas. [Internet]. Disponible en: http://agroecuador.org/images/pdfs/buenas-practicas/pec/Manuales-de-aplicabilidad-deBP-Avicolas.pdf

22. Walpole R, Myers $R$, Myers S, Ye K. 2012. Probabilidad y estadística para ingeniería y ciencias. $9^{a}$. ed. México: Pearson. 816 p. 